\title{
Spinal Cord Editor's Page January 2008
}

From the editorial office we wish you a happy and prosperous 2008.

Spinal Cord has increased its pagination for 2008. This decision was made to enable a higher number of submissions to be accepted and to shorten the time before articles appear in print.

In this issue, a number of papers relate to one topic: pain.

In their contribution, Sawatzki, Bishop and Miller review psychometric properties of pain measures specifically used in spinal cord injury (SCI) populations and give recommendations for improvement.

Philip Siddall (in Chapter 66 of Wall and Melzack's Textbook of Pain, 5th edition, edited by S McMahon and M Kotzenburg, published by Elsevier, 2006) states that pain is a frequent consequence of SCI affecting two-thirds of people with SCI. Pain has a significant impact on the patient, the relatives, the treating physicians and other health carers. Pain can be musculoskeletal, visceral and neuropathic - the latter rated the most severe.

Quoting P Siddall: 'Recent studies have provided evidence that suggests that neuropathic SCI pain may be due to abnormal neuronal activity at a number of levels, including nerve roots, the spinal cord close to the site of the injury and brain structures. Attempts at regeneration, loss of inhibition and increased excitatory influences may all result in the presence of a population of neurons that have an increased excitability leading to the generation and amplification of nociceptive signals'. All involved in the management of pain in SCI patients know how difficult the treatment is and how satisfactory results are not easily obtained, be it with pharmacologic treatment, anaesthetic blockade, stimulation techniques or surgical procedures. It goes without saying that a combination of physical treatment and psychological treatment is very important. Pain is such a huge subject and a fair knowledge is necessary to be able to properly care for patients with chronic pain. Plasticity of pain, representation of pain in the brain, development of pain pathways, central pain modulation are just a few examples of pain knowledge. It is not surprising that there has been a growth in the number of specific pain consultations and clinics over the last few years.

Many interesting manuscripts on different topics can be found in this first issue of 2008. Enjoy reading.

Spinal Cord (2008) 46, 1; doi:10.1038/sj.sc.3102156

JJ Wyndaele

Editor

E-mail: spinalcord@uza.be 